\title{
El vaciamiento que antecede la renovación. Reflexiones a partir del proyecto del distrito gubernamental en el sur de la ciudad de Buenos Aires
}

The Hollowing Out that Precedes the Urban

Renewal. Reflections From the Project of the Civic

Center in the South of Buenos Aires city

O esvaziamento que antecede a renovação.

Reflexões a partir do projeto do distrito governamental

no sul da cidade de Buenos Aires

Carolina Gonzalez Redondo*

Recibido: 12 de septiembre de 2018

Aprobado: 10 de enero de 2019

Doi: http://dx.doi.org/10.12804/revistas.urosario.edu.co/territorios/a.7228

\section{Para citar este artículo:}

Gonzalez Redondo, C. (2020). El vaciamiento que antecede la renovación. Reflexiones a partir del proyecto del distrito gubernamental en el sur de la ciudad de Buenos Aires. Territorios(42), 1-22. Doi: http://dx.doi. org/10.12804/revistas.urosario.edu.co/territorios/a.7228

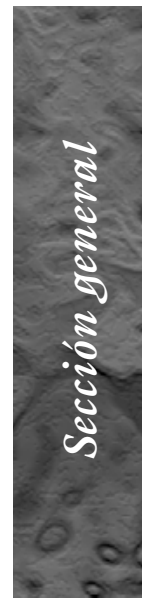

* Becaria doctoral CONICET con sede en el Instituto de Investigaciones Gino Germani. Correo electrónico:carito_gr@yahoo.com. ar / carolina.gredondo@ gmail.com ORCID: https:// orcid.org/0000-00028338-8148 
Palabras clave

Vacio urbano, renovación urbana, distrito gubernamental, análisis del discurso, Buenos Aires.

Keywords

Urban vacuum, urban renewal, civic center, discourse analysis, Buenos Aires city.

Palavras-chave

Vazio urbano, renovação urbana, distrito governamental, análise do discurso, Buenos Aires.

tersitarias 42

\section{RESUMEN}

Este artículo reflexiona sobre el proceso de legitimación previo al despliegue de políticas de renovación urbana en el sur de la ciudad de Buenos Aires. Para ello, analiza el proceso de vaciamiento simbólico y material de un predio del barrio Barracas, en el que se proyectaba la construcción del distrito gubernamental, un desarrollo inmobiliario en el que se instalarían oficinas del gobierno local, junto con emprendimientos comerciales. En este sentido, este artículo dialoga con otros trabajos que estudian la producción de vacíos urbanos como parte de las dinámicas capitalistas de producción de ciudad, analizando especialmente cómo este se produce discursivamente y cómo la noción de renovación urbana emerge como su par asociado, su respuesta necesaria e indiscutible.

\section{ABSTRACT}

This article reflects the process of legitimization prior to the deployment of urban renewal policies in the south of Buenos Aires. It analyzes the process of symbolic and material hollowing out of a property in the neighborhood of Barracas, in which authorities planned the construction of the civic center. In this sense, this paper dialogues with other works that study the production of urban vacuum as part of the capitalist dynamics of city production. It especially analyses how discourse produces it and how the notion of urban renewal emerges as its associated pair, as its necessary and indisputable answer.

\section{RESUMO}

Este artigo reflete sobre o processo de legitimação prévio à implementação de políticas de renovação urbana no sul da cidade de Buenos Aires. Para isto analisa o processo de esvaziamento simbólico e material de um prédio do bairro de Barracas, no qual se instalariam escritórios do governo local, junto com empreendimentos comerciais. Neste sentido, este artigo dialoga com outros trabalhos que estudam a produção de vazios urbanos como parte das dinâmicas capitalistas de produção de cidade, analisando especialmente como se produz discursivamente um vazio urbano e como a noção de renovação urbana emerge como seu par associado, como sua resposta necessária e indiscutível. 


\section{Introducción}

Este artículo reflexiona sobre el proceso de legitimación previo al despliegue de políticas de renovación urbana en el sur de la ciudad de Buenos Aires. Para ello analiza el proceso de vaciamiento simbólico y material de un predio de 40 hectáreas, ubicado en el barrio de Barracas, en el que se proyectaba la construcción del distrito gubernamental, un desarrollo inmobiliario en el que se instalarían oficinas del Gobierno de la Ciudad de Buenos Aires (GCBA), junto con locales comerciales. En este sentido, este artículo dialoga con otros trabajos que estudian la producción de vacíos urbanos como parte de las dinámicas capitalistas de producción de ciudad (Franquesa, 2007; Marcús, Aquino \& Vázquez, 2016; Marcús \& Vázquez, 2017; Stanchieri \& Aricó, 2013), analizando especialmente cómo se construye discursivamente la noción de vacio urbano.

El distrito gubernamental o centro cívico $^{1}$ es una política que impulsa el GCBA desde el año 2008, en el marco de un conjunto de iniciativas orientadas a la renovación de la zona sur de la ciudad. Esta propuesta - al igual que otras que proliferan en las ciudades latinoamericanas- se enmarca en el denominado urbanismo neoliberal (Pradilla Cobos, 2009; Jaramillo, 2014) que se consolidó a partir de los años 80 con la reconfiguración del modelo de producción capitalista. Este urbanismo neoliberal, en lugar de regular el mercado inmobiliario, se pliega a

EL VACIAMIENTO QUE ANTECEDE LA RENOVACIÓN su dinámica generando las condiciones para una inversión segura, en consonancia con un tipo de gestión urbana (el empresarialismo urbano), en el que prima una visión empresarial sobre la ciudad y sobre lo público (Harvey, 1989).

El concepto de empresarialismo urbano caracteriza a un tipo de gestión que "descansa más en una asociación público-privada focalizada en inversión y desarrollo económico con una construcción especulativa del lugar, que en el mejoramiento de las condiciones del territorio" (Harvey, 1989, p. 89). Así el Estado adquiere un rol de facilitador y promotor de la actividad económica, asumiendo los riesgos y garantizando la rentabilidad del capital privado. Tal como apuntan Di Virgilio y Guevara (2014, p. 14), "el resultado en términos urbanísticos es por demás deficitario, porque el capital privado actúa de manera individual y fragmentada, poniendo de relieve las contradicciones de la urbanización capitalista".

De este modo, el urbanismo neoliberal profundiza y acentúa el desarrollo geográfico desigual (Harvey, 1989), inherente al sistema capitalista, acelerando los ritmos de la destrucción creativa (Franquesa, 2007). Siguiendo a Theodore, Peck y Brenner $(2009$, p. 6), este proceso combina dos momentos - "dialécticamente entrelazados, pero analíticamente diferenciables" - de destrucción parcial de estructuras institucionales heredadas, por un lado, y de creación tendencial de una nueva infraestructura de crecimiento
${ }^{1}$ Esta política recibió distintos nombres. En este trabajo utilizamos alternadamente las denominaciones "distrito gubernamental" o "centro cívico” como sinónimos. Con "Parque Cívico" nos referimos al primer antecedente del proyecto del año 2008 y reservamos el término "nueva sede de gobierno" para aludir a las nuevas oficinas del GCBA situadas en Parque Patricios y en La Boca.

tersitarios 42

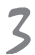


2 Tal como plantean estos autores, la producción de vacios urbanos es una dinámica constitutiva de la producción del espacio urbano en el contexto neoliberal y en este sentido el caso que aqui analizamos del barrio de Barracas no resulta una excepción, sino que es parte de una lógica más amplia que se da en otras zonas de la ciudad de Buenos Aires. Así, por ejemplo, Marcús y Vázquez (2017) analizan los casos del playón ferroviario del barrio de Caballito y la manzana 66 en el barrio de Balvanera. Ambos procesos implicaron un vaciamiento material y simbólico de prácticas y significados para dar lugar a la presentación de proyectos de (re) llenado rentables para el capital inmobiliario que, producto de la oposición de organizaciones vecinales, se vieron interrumpidos.

${ }^{3}$ Hasta el año 1996, la ciudad de Buenos Aires revestía el carácter de Capital Federal. Con la nueva Constitución Nacional de 1994, se establece el proceso de autonomización de la ciudad que se implementa a partir de 1996. Entonces se conforma Buenos Aires como un distrito autónomo, similar a la categoría de las provincias, con su propia estructura de poderes: ejecutivo, legislativo y judi-

tersitarios 42 económico orientada al mercado, por el otro. Con relación al espacio, la destrucción creativa define el proceso mediante el cual los lugares son destruidos y creados (devaluados y revalorizados) para garantizar la acumulación capitalista. Para que exista una diferencia potencial de renta (rentgap), es preciso que se produzca un ciclo de desvalorización (de destrucción del valor) que genere las condiciones económicas objetivas para su revalorización (Smith, 2012).

Esta lógica de obtención de beneficios económicos guía las actuales políticas de renovación urbana que proliferan —con matices y diversas temporalidades - en las ciudades latinoamericanas, en el marco de los crecientes procesos de mercantilización del desarrollo urbano (de Mattos, 2008) y de valorización del suelo (Pradilla Cobos \& Márquez López, 2016). Estas políticas de renovación urbana - entendidas como las acciones e inversiones tendientes a transformar y mejorar material y discursivamente el entorno urbanístico de un área determinada (Díaz Parra, 2013; Rodríguez, 2015)— suelen ser concebidas para espacios urbanos de diferentes tamaños (desde un predio o una manzana, hasta barrios completos) que previamente son construidos simbólica y materialmente como deteriorados, abandonados o incluso vacantes. Dicho en otros términos la contracara de la renovación es - como analizaremos en este artículo- el proceso previo de desinversión, deterioro y abandono que da lugar a la caracterización de un espacio como "vacío" de prácticas, usos y sentidos.

Un conjunto de trabajos ha analizado la producción de vacios urbanos como requisito que antecede las propuestas de renovación (Franquesa, 2007; Marcús, Aquino \& Vázquez, 2016; Marcús \& Vazquez, 2017; Stanchieri \& Aricó, 2013). ${ }^{2}$ En sintonía con ellos, este artículo se centra en las condiciones de posibilidad de una política de renovación urbana: el distrito gubernamental, proyectada en un predio del barrio de Barracas, al sur de la ciudad de Buenos Aires. Para ello, posamos la mirada en el primer momento de una política pública; es decir, en la transformación de un asunto social en una cuestión (Oszlak \& O’Donnell, 1981). Esto resulta importante porque el modo en el que es conceptualizada la cuestión incide en las respuestas que se formularán para su resolución. En este sentido, a lo largo de este artículo nos preguntamos cómo es definida la cuestión que da lugar al centro cívico en este espacio.

A partir del análisis discursivo de una serie de documentos de diagnóstico y planificación, advertimos que el predio donde se proyectaba el distrito gubernamental fue objeto de un proceso de vaciamiento simbólico al ser tematizado como un "espacio vacío", que se completa con un vaciamiento material a partir de la acción y omisión del ejecutivo local. Siguiendo a Marcús y Vazquez (2017), el proceso activo de construcción simbólica y material de un vacío urbano constituye 
la precondición para la presentación de los proyectos de (re)llenado - en este caso el distrito gubernamental- como necesarios y deseables. Nuestro abordaje desde el análisis de los discursos permite precisamente echar luz sobre esto último, focalizando en el proceso de vaciamiento simbólico. Así, advertimos cómo se produce discursivamente un vacío urbano y cómo la noción de renovación urbana va emergiendo como su par asociado, como su respuesta necesaria e indiscutible.

\section{Consideraciones metodológicas}

Nos apoyamos en una estrategia metodológica cualitativa que combina diversos tipos de análisis. Por un lado, a través del relevamiento bibliográfico, la sistematización de fuentes documentales y de entrevistas a informantes clave reconstruimos el contexto de emergencia de la propuesta del distrito gubernamental. Esto nos lleva a profundizar en el proceso histórico de urbanización de Buenos Aires, las políticas de renovación más recientes orientadas al sur de la ciudad y las políticas públicas de atención a la salud mental. Por otro lado, realizamos un análisis discursivo de un corpus conformado por discursos sobre la ciudad de Buenos Aires, especialmente sobre el sector bajo estudio, que, al ser seleccionados mediante mecanismos sociales e institucionales específicos, constituyen documentos (Narvaja de Arnoux, 2009).

Conforman nuestro corpus aquellos documentos oficiales de diagnóstico y planificación elaborados luego del retorno de la democracia por el GCBA - o en su defecto la Municipalidad de la Ciudad de Buenos Aires $\left(\mathrm{MCBA}^{3}\right)$ - en conjunto con distintas instituciones ligadas al urbanismo y la arquitectura ${ }^{4}$ en los que se caracteriza al barrio de Barracas y al predio en cuestión. Este recorte nos lleva a considerar seis documentos, tomando como punto inicial la publicación de 1988 que reúne los proyectos ganadores del concurso Ideas urbano-arquitectónicas para Buenos Aires - en adelante " 20 ideas"- producto de un convenio de cooperación entre la MCBA y la Comunidad de Madrid. ${ }^{5}$ La mirada experta y oficial que se combina en estos documentos - la dimensión del espacio concebido, en términos de Lefebvre (2013) - nos permite reflexionar sobre el carácter performativo de estos discursos. Como veremos, al proponer una representación "legítima" sobre la ciudad, sugieren asimismo una interpretación y buscan orientar la acción sobre ella. De ahí su importancia y validez para rastrear en ellos cómo es definida la cuestión que da lugar al distrito gubernamental.

Para su abordaje, construimos un dispositivo para el análisis de los discursos que, siguiendo la perspectiva teórica de la escuela francesa, combina elementos de las teorías de la enunciación y la argumentación. ${ }^{6}$ Por un lado, el análisis de ciertos recursos lingüísticos y operaciones enunciativas nos permite identificar cómo se construye a sí mismo el enunciador - con qué ethos ${ }^{7}$ se asocia- y qué enunciatario
$\Longleftarrow$

cial. En lo que respecta al poder ejecutivo, la entonces Municipalidad de la Ciudad de Buenos Aires se convirtió en el Gobierno de la Ciudad de Buenos Aires.

${ }^{4}$ Entre ellas, la Facultad de Arquitectura y Urbanismo de la UBA (FADU), la SCA, el Consejo Profesional de Arquitectura y Urbanismo (CPAU), el Centro Argentino de Ingenieros (CAI) y el Consejo Profesional de Ingeniería Civil (CPIC).

5 Siguiendo a Jajamovich (2012), este concurso -que supuso la articulación entre redes de técnicos $y$ profesionales españoles $y$ locales - marca el fortalecimiento de la perspectiva de los arquitectos proyectistas por sobre la mirada de planificadores urbanose investigadores vinculados a las ciencias sociales, quienes hasta entonces se disputaban la capacidad de definir los problemas de la ciudad $y$ las maneras "adecuadas" de resolverlos. Junto con esto, implica además la consolidación del modelo de intervención fragmentaria por proyectos, que predominará en adelante frente al paradigma de la planificación urbana regional, begemónico en décadas anteriores (Gorelik, 2004; Jajamovich, 2012). De abi que lo tomemos como punto de inicio de nuestro recorte.

territarios 42 
$\Longleftarrow$

${ }^{6}$ En los primeros trabajos de la escuela francesa de análisis del discurso predominó un esfuerzo por articular las teorias del discurso, la ideología y el psicoanálisis con Pecheux comogran exponente (Charaudeau \& Maingueneau, 2005). Hacia fines de la década del 70, su foco de estudio se trasladó hacia la enunciación y posteriormente a la argumentación (Montero, 2014). Retomamos esta perspectiva porque invita a pensar en la dimensión conflictiva que constituye todo discurso en tanto que su sentido no es unívoco, existe una lucha por su fijación. No bay una "democracia discursiva", no cualquier voz es legítima en cualquier momento y lugar. Las tematizaciones no son aleatorias, sino que responden a tendencias begemónicas y leyes tácitas que regulan lo posible de ser dicho en un momento dado (Angenot, 2010).

7 Este término remite al tono y al cuerpo que construye el enunciador a lo largo del enunciado. Ese ethos juega el papel de garante de la enunciación y su identificación a través del análisis permite advertir el posicionamiento del enunciador, el lugar social desde el que habla, el enunciatario que cons-

territarias 42 configura. Por el otro, el análisis de las estrategias argumentativas, el despliegue de topos retóricos y lugares comunes (Amossy, 2000) nos ayuda a deconstruir las tematizaciones sobre el espacio en cuestión. Así, nuestro análisis se asienta en el nivel intradiscursivo, ese plano en el que se sitúan las operaciones enunciativas y argumentativas, donde el sujeto-enunciador aparece incorporando de distintos modos las voces ajenas y administrando la polifonía (Montero, 2014). Sin embargo, tiene como horizonte su vínculo con el interdiscurso, ese nivel constitutivo en el que el sujeto es hablado por el discurso, es un sujeto del inconsciente atrapado por las formaciones discursivas e ideológicas. ${ }^{8}$

\section{La renovación del sur de Buenos Aires, una mirada histórica}

El distrito gubernamental, junto con otras políticas dirigidas al área sur de la ciudad se presentan como una nueva respuesta a una cuestión (Oszlak \& O’Donnell, 1981) que se ha ido construyendo como tal a lo largo del proceso histórico de urbanización de Buenos Aires: el "deterioro" de la zona sur y su "desequilibrio" con respecto al norte. ${ }^{9}$ Ahora bien, ¿̇en qué consisten ese "deterioro" y ese "desequilibrio"? Por un lado, el "desequilibrio" expresa más bien una gran desigualdad en las condiciones de vida de las personas que habitan a uno y otro lado de la Av. Rivadavia ${ }^{10}$ (ver mapa 1). De hecho, es en las comunas ${ }^{11}$ del sur de la ciudad (4 y 8 ) donde se concentran los valores más críticos en relación con indicadores socioeconómicos y habitacionales. ${ }^{12}$ De manera complementaria, el "deterioro" que aparece en los diagnósticos oficiales nos remite al espacio construido: las instalaciones industriales y galpones en desuso, los grandes espacios verdes sin mantenimiento, los grandes equipamientos urbanos y conjuntos habitacionales relegados, las trazas de autopistas y de trenes que funcionan como "barreras urbanas", etc.

Este diagnóstico, que tiende a presentarse como algo dado, es resultado por lo contrario de un proceso histórico de desarrollo desigual de esta ciudad, que guarda relación con la historia de su urbanización y sus diferentes lógicas (Pírez, 1995). La falta de interés por parte del mercado, la acción y omisión por parte del Estado y las acciones de la población de menores recursos tendientes a garantizarse un espacio en la ciudad explican en gran parte las características socio-territoriales actuales de la zona sur (Gonzalez Redondo, 2018). ${ }^{13}$ Los urbanistas, técnicos y funcionarios públicos de cada momento no ignoraron esta situación y así fue como el desarrollo desigual de la ciudad empezó a ser pensado muy tempranamente como una cuestión a resolver (Oszlak \& O'Donnell, 1981).

Si bien este "desequilibrio" se reconoce desde fines del siglo XIX, advertimos que las políticas urbanas de la dictadura constituyen una bisagra, en la medida en que dejaron profundas huellas en el tejido 
urbano que prepararon las condiciones para el proceso de renovación posterior. Así, el neoliberalismo realmente existente (Theodore, Peck \& Brenner, 2009) en la ciudad de Buenos Aires se fue configurando desde mediados de la década del 70 a través de un proceso de sedimentación y superposición de diversos planes y proyectos urbanos, los sucesivos ajustes del aparato burocrático del Estado y las distintas políticas urbanas, orientadas por concepciones afines al empresarialismo urbano, tal como se definió más arriba (Harvey, 1989). A lo largo de este proceso, se reconocen diversos matices, temporalidades y reacomodamientos en función de los conflictos que se van suscitando, pero se mantiene como denominador común el rol activo del Estado para propiciar las transformaciones urbanas en curso.

Desde su autonomización política, en 1996, las políticas hacia el sur de la ciudad se orientaron a "incorporar el sur al norte" (Rodríguez \& Di Virgilio, 2014); el Estado condujo un proceso activo de renovación urbana que tiene su correlato en la progresiva concentración del gasto público en este área, particularmente en las comunas 4 y 8 (Di Virgilio \& Guevara, 2014; Guevara, 2010; Rodríguez, Bañuelos \& Mera, 2008). Las políticas de renovación estuvieron primero concentradas en las áreas más cercanas al microcentro porteño, luego se fueron extendiendo hacia el sudeste, abarcando los barrios de La Boca y Barracas y más recientemente llegaron a los barrios de

EL VACIAMIENTO QUE ANTECEDE LA RENOVACIÓN la comuna 8 , pasando previamente por Parque Patricios. ${ }^{14}$

En este contexto, a partir del año 2008 , el GCBA proyectó la creación de seis distritos especializados que, al desarrollarse de manera sistemática, rompen con la lógica fragmentaria de las intervenciones urbanas precedentes (Goicoechea, 2016), a la vez que profundizan y extienden el proceso de renovación de las comunas 4 y 8 . Por un lado, se crean cinco distritos económicos (DE) que constituyen áreas específicas en las que se promueve la radicación de empresas e instituciones de un mismo sector económico, mediante exenciones impositivas, créditos blandos y la creación de infraestructura, entre otras cosas (figura 1). De manera complementaria, se formula el proyecto del distrito gubernamental, que tiene la particularidad de relocalizar el sector público (en lugar de empresas privadas), en un contexto barrial donde se están impulsando otras iniciativas de revalorización del espacio urbano.

Cinco de estos seis distritos especializados (incluyendo el gubernamental) se proyectaron en los barrios del sur de la ciudad (mapa 1) y parte de su justificación radica en la necesidad de "integrar la ciudad" y para ello "desarrollar su zona sur". Así, junto con una cantidad de iniciativas de desarrollo urbano público-privadas, ${ }^{15}$ estos distritos se presentan como una nueva respuesta al problema público (Subirats et al., 2008) del "deterioro del sur". truye y los ámbitos institucionales que legitiman su enunciado, en asociación con una determinada formación discursiva (Maingueneau, 2008).

- Acuñado por Foucault (2002) y reformulado por Pecheux (1971), el concepto de formación discursiva refiere a "lo posible de ser dicho desde una posición dada en una coyuntura dada" (Pecheux, 1971 citado en Maldidier, 1990, p. 206). Las palabras cambian de sentido al pasar de una formación discursiva a otra. La formación discursiva sólo se mantiene y se constituye a través del interdiscurso (Maingueneau, 2008).

${ }^{9}$ Las nociones de "deterioro de la zona sur" y "desequilibrio norte-sur" constituyen categorias nativas que refieren a las diferencias y desigualdades territoriales entre las zonas norte y sur de la ciudad, limite que se configura física y simbólicamente con la traza de la Av. Rivadavia. Este diagnóstico de una zona sur "deteriorada" $y$ de un "desequilibrio entre norte y sur" aparece recurrentemente en los diferentes documentos de diagnóstico y planificación de la ciudad de Buenos Aires al menos desde comienzos del siglo XX. Para profundizar

territarios 42 
$\Longleftarrow$

en este tema ver Romero (2009), Gorelik (1998), Goicoechea (2016) y Gonzalez Redondo (2018).

${ }^{10}$ Tal como se observa en el mapa 1, la Av. Rivadavia es el límite físico y simbólico entre el norte y el sur de la ciudad.

11 Las 15 comunas constituyen un nuevo nivel de gestión politico-administrativo de la ciudad de Buenos Aires desde el año 2005, en que se sancionó la ley 1777 .

12 Para un análisis detallado ver CEyS (2013) y Gonzalez Redondo (2018).

${ }^{13}$ A lo largo del proceso de urbanización de la ciudad de Buenos Aires, la zona sur se fue consolidando como un área de perfil industrial, relativamente poco interesante para los desarrolladores privados, debido al carácter inundable de sus tierras y a su nivel de contaminación ambiental. El Estado contribuyó a generar este desinterés a través de la falta de inversión pública, la sanción de determinada normativa y la instalación de equipamientos "indeseables" como los basurales. Asimismo, es en esta zona de la ciudad donde se fue concentrando la mayor cantidad de población vulnerable, que conformó las denominadas

\section{territarias 42}

Figura 1. Ubicación de los distritos especializados y de las nuevas sedes de gobierno

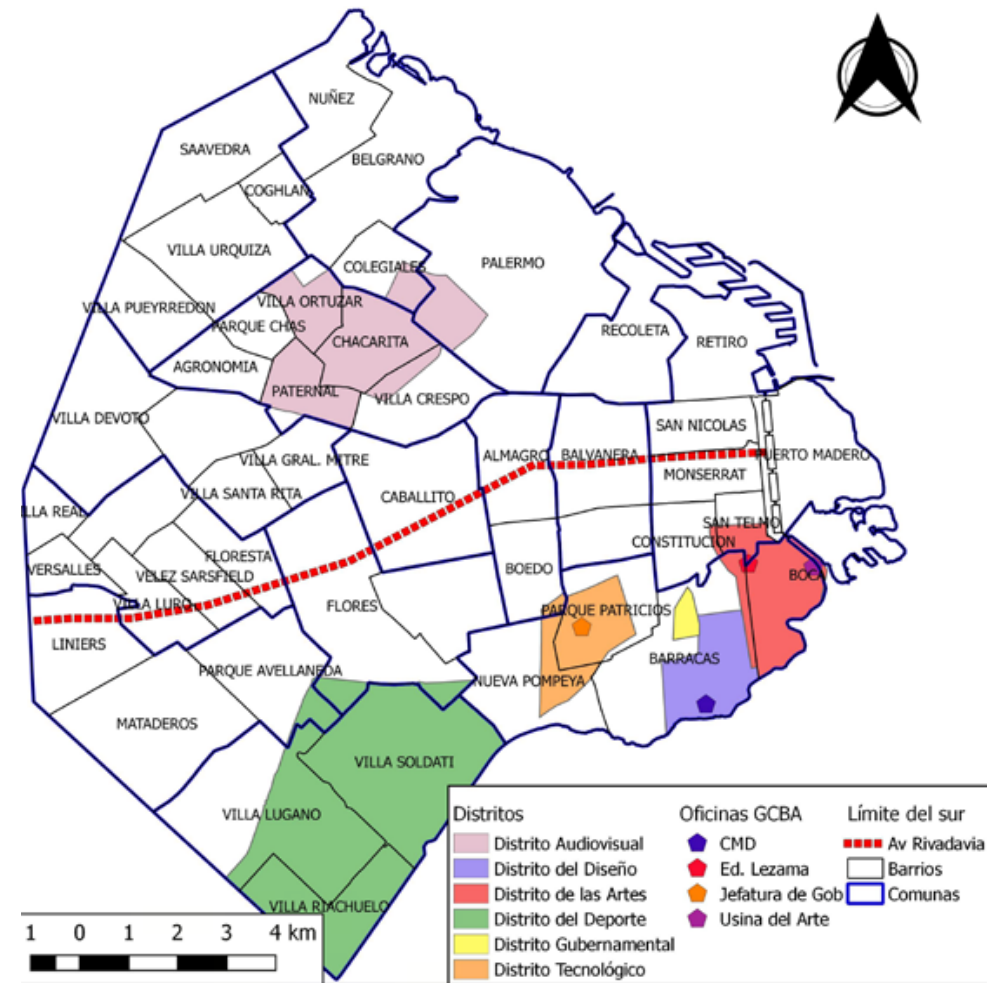

Fuente: elaboración propia con base en información del GCBA.

En el año 2008, el GCBA junto con la Sociedad Central de Arquitectos (SCA) llamó a un concurso para el desarrollo del Parque Cívico, un lugar donde se ubicarían las funciones administrativas del GCBA junto con locales comerciales. La propuesta ganadora transformaba el predio completo y presuponía el cierre de los tres hospitales psicoasistenciales que allí funcionan — Borda, Tobar García y Moyano- (imagen 1). ${ }^{16}$ Esta iniciativa no pasó de la instancia proyectual. Sin embargo, en el año 2012, se llamó a concurso y licitó la construcción de los nuevos edificios del Distrito Gubernamental, que constituyen la fase I del proyecto del Parque Cívico. Estos edificios serían construidos en una parte del predio, se trata de un sector dentro del Hospital Borda comprendido entre las calles Av. Amancio Alcorta y Pedriel y las líneas divisorias indicadas en el plano (imagen 2). 
Imágenes 1 (izq.) y 2 (der.). Diseño del Parque Cívico según la propuesta ganadora del concurso de 2008 (izq.). Plano de localización del área destinada a la primera etapa del distrito gubernamental, zona grisada (der.)
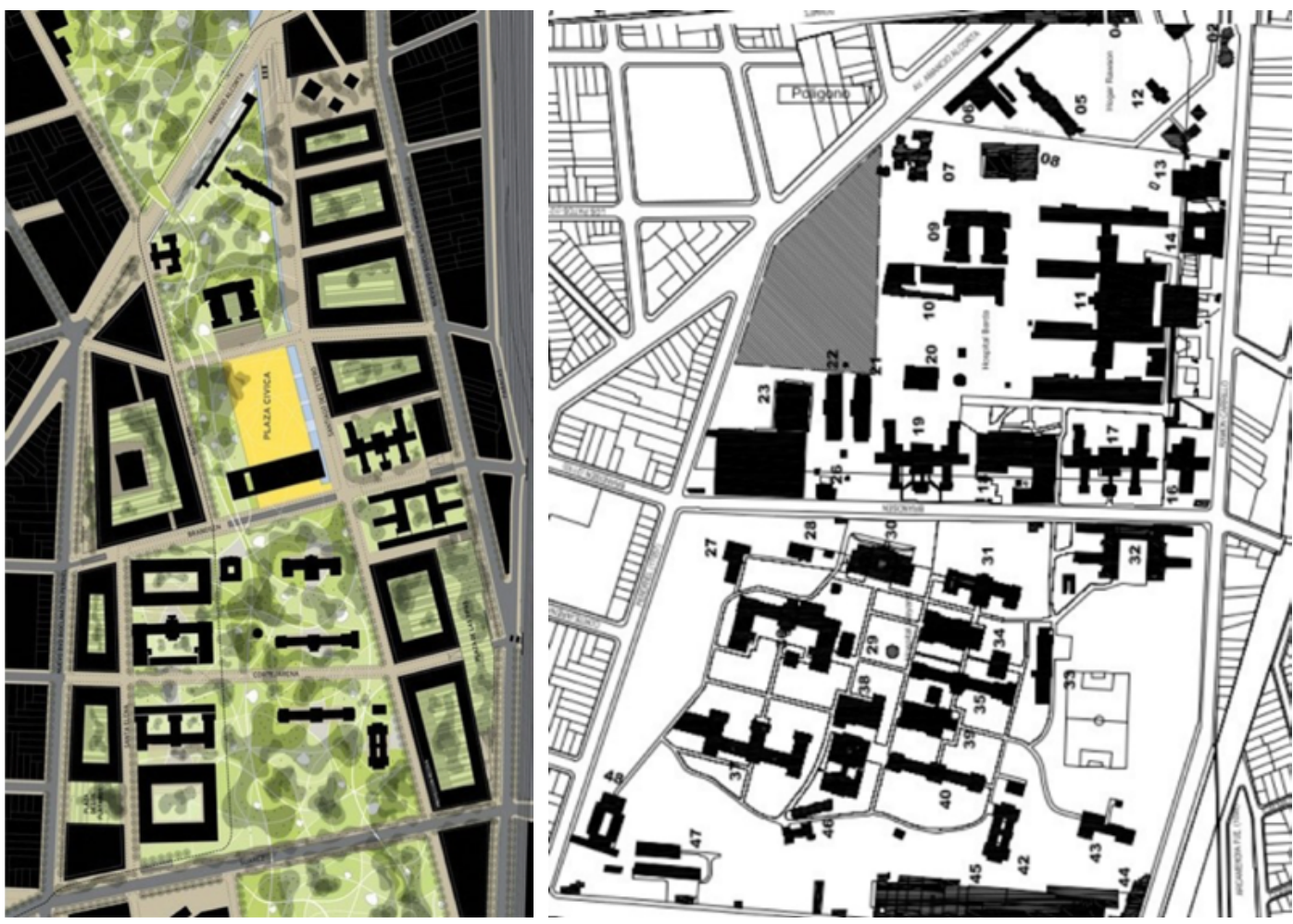

Fuente: Bases Concurso Nuevos Edificios del Distrito Gubernamental, SCA, 2011

El distrito gubernamental implicaba entonces la afección de una parte del predio del Hospital Borda, donde funcionaba el taller protegido 19 y una cancha de fútbol de uso de los pacientes. Este espacio se torna objeto de disputa entre el GCBA y un conjunto de actores que se oponen a la propuesta. A partir de entonces, se abre un proceso sinuoso de implementación de la política, que tiene su

epicentro el 26 de abril de 2013, cuando las fuerzas policiales reprimen a trabajadores, pacientes y legisladores dentro del Hospital Borda. Este hecho, junto con otros factores, incidió para que el GCBA se reposicionara y mudara sus oficinas a otros edificios ubicados también en la zona sur de la ciudad (mapa 1). De este modo, quedó suspendida - al menos, por el momento- la construcción del distrito
$\Longleftarrow$

villas de emergencia, algunas de las cuales surgieron a partir de politicas de relocalización y construcción de vivienda transitoria, otras por asentamiento espontáneo y otras por la conjunción de ambos procesos (Goicoechea, 2016). Para mayor detalle sobre el proceso de urbanización desigual de Buenos Aires ver Scobie (1977), Romero (2009), Arqueros Mejica et al., (2011); Oszlak (1983; 2017); Jajamovich y Menazzi (2012); Rodríguez y di Virgilio (2014); Silvestriy Gorelik (2000); Gonzalez Redondo (2018).

${ }^{14}$ Para profundizar sobre los procesos de renovación en la zona sur de la ciudad durante las décadas del 90 y el 2000, consultar Herzer (2008) y di Virgilio et al. (2011).

${ }^{15}$ La construcción de institutos terciarios de la $U B A$, la extensión de la línea $H$ del subterráneo, la creación de lineas de Metrobus, la inauguración del predio para recitales "Ciudad Rock", entre otros. Ver nota de prensa del GCBA, "Acciones para el crecimiento de los barrios del Sur", disponible en http:// www.buenosaires.gob.ar/ noticias/el-sur-prioridadestrategica

${ }^{16}$ A lo largo de este trabajo nos referiremos a este espa-

territarios 42 
$\Leftarrow$

cio como predio o polígono de los hospitales.

${ }^{17}$ Un conjunto de actores -entre los que se destacan los trabajadores nucleados en la Asociación de Trabajadores del Estado (ATE), organizaciones sociales $y$ algunos legisladores de la oposición-desplegó una serie de estrategias para obturar el desarrollo del distrito gubernamental en este predio. Entre ellas se destacan la movilización, la difusión, la presentación de un proyecto alternativo para refuncionalizar el predio, la articulación con el poder legislativo y la judicialización del conflicto. Estas estrategias en conjunto, particularmente la medida cautelar de fines de 2012 que probibió el comienzo de las obras junto con la represión a pacientes y trabajadores dentro del hospital, fueron cruciales para que se interrumpiera el proyecto del distrito gubernamental. Así, en $2015 \mathrm{el}$ GCBA decidió mudar sus oficinas de gobierno a otros dos edificios de la zona sur de la ciudad, uno ubicado frente al Parque Patricios, en el corazón del distrito tecnológico, y el otro frente al Parque Lezama, dentro del distrito de las artes. Para profundizar sobre el conflicto generado a par-

territarias 42 10 gubernamental en dicho lugar ${ }^{17}$ (Gonzalez Redondo, 2018).

Sin embargo, cabe preguntarse qué hizo posible que se proyectara el distrito gubernamental en este predio donde funcionaban hospitales públicos de atención a la salud mental. Aquí cobra relevancia el cruce de esta política del distrito gubernamental con otra cuestión en agenda (Oszlak \& O’Donnell, 1981): la reconversión del sistema de salud mental. En el marco de un cambio normativo nacional y local que insta a la sustitución de los efectores monovalentes, como es el Hospital Borda, por dispositivos comunitarios y ambulatorios, ${ }^{18}$ el ejecutivo local desplegó un proceso de progresivo vaciamiento material del sistema de salud mental, en general, y del Hospital Borda, en particular, que si bien es de larga data, se profundizó a partir de las gestiones del $\mathrm{PRO}^{19}$ en el GCBA.

Este vaciamiento material se sostiene en el progresivo abandono y deterioro del sistema público de salud mental de la ciudad, que entre sus principales problemas incluye: dificultades en la provisión de insumos; un creciente deterioro de las condiciones de infraestructura; el incumplimiento de los principales lineamientos de las nuevas normativas, y grandes carencias en lo que respecta a recursos humanos (CESLSM, 2010, 2012; 2014-2015). Esto a su vez se complementa con una progresiva disminución en la cantidad de pacientes internados debido, entre otros motivos, a su derivación a través de las obras sociales a clínicas de internación privadas, que tampoco se adecúan al nuevo paradigma de salud mental (CESLSM, 2010, 2012; 2014-2015). ${ }^{20}$ Este proceso de destrucción creativa (Theodore, Peck \& Brenner, 2009), que desarticula las estructuras e institucionalidades heredadas para crear las condiciones para la construcción de una nueva infraestructura (el distrito gubernamental), se apoya en un proceso previo de vaciamiento simbólico del predio en cuestión, que analizamos a continuación.

\section{La construcción de un espacio vacio}

Tanto el predio de los hospitales como el barrio que lo alberga, Barracas, han sido objeto de diagnóstico y de propuestas en diversos documentos elaborados por el GCBA en convenio con distintas instituciones ligadas al urbanismo y la arquitectura. En este apartado, analizamos cómo es caracterizado dicho predio y su entorno inmediato en estos documentos. Buscamos trazar algunas pistas sobre cómo ese espacio es tematizado y problematizado como un espacio vacio, construcción que, como veremos, constituye una condición de posibilidad para que se proyecte allí el distrito gubernamental.

En estos documentos aparece en forma recurrente la expresión vacío urbano (o sustitutos como "espacio vacío”, "espacio vacante" o "área deteriorada”) para caracterizar un predio que lejos de estar vacío alberga funciones públicas vinculadas a la atención de la salud mental. Esta noción 
de vacio urbano se construye como objeto discursivo en los diferentes documentos. Siguiendo a Narvaja de Arnoux (2009), ${ }^{21}$ la repetición de lexemas (y sus sustitutos), la insistencia en determinados atributos, la oposición con otros objetos y los diversos modos de reformulación permiten identificar en el nivel intradiscursivo la conformación de este objeto discursivo. Ahora bien, las condiciones de aparición de ese objeto discursivo son históricas, exceden al sujeto-enunciador, en la medida en que la secuencia discursiva está dominada por una formación discursiva determinada (Narvaja de Arnoux, 2009).

En los documentos que analizamos, el objeto discursivo vacio urbano, que remite a una formación discursiva específica vinculada a las disciplinas de la arquitectura y del urbanismo, no presenta un significado estable ni unívoco. Por el contrario, sus distintas formulaciones en el intradiscurso suponen diversos efectos de sentido en relación con lo que "debe hacerse" con ese tipo de espacios y particularmente con el predio de los hospitales. Los distintos centramientos en cada documento irán definiendo la cuestión que posteriormente dará lugar a la emergencia del distrito gubernamental como una propuesta "necesaria" para dicho espacio.

\section{La recuperación de un pasado perdido}

Los dos documentos que analizamos en esta sección -Ideas urbano-arquitectónicas para Buenos Aires" o "20 ideas"
$(1988)^{22}$ y "Barracas. Diagnóstico y estrategias"23 (1992)— son de finales del siglo pasado y tienen aspectos en común, que guardan relación con su contexto histórico de producción. ${ }^{24}$ En ese momento una de las discusiones centrales del urbanismo en Argentina es la reformulación del planeamiento y la participación ciudadana en el nuevo contexto democrático (Jajamovich, 2012). En este sentido, no llama la atención que en ambos casos identifiquemos un enunciador que se posiciona en el rol de funcionario público, que hace uso de un tono reflexivo y valoriza el "diálogo" y "el debate". Así, el garante del discurso -que a su vez le da legitimidad a los diagnósticos y propuestas de cada documento- es la democracia y el debate que ella habilita para marcar una ruptura con el pasado dictatorial.

Pero también el discurso científico, como discurso constituyente (Maingueneau \& Cossutta, 1995), funciona como garante en la medida en que son en primer lugar "los profesionales" y "especialistas" quienes desde su rol de expertos forman parte de este debate. En este sentido, el rol del político se combina con un enunciador asociado a un ethos de planificador y de experto, que demuestra competencia sobre la materia y le habla a un enunciatario par, capaz de comprender el vocabulario técnico que se pone en juego.

Desde este posicionamiento de autoridad del enunciador, se tematizan la zona sur, el barrio de Barracas y el predio de los hospitales. Tanto Barracas como "el sur" de esta politica, consultar González Redondo (2015; 2018).

${ }^{18}$ Desde hace varias décadas, tanto a nivel inter nacional como local, se ha instalado el debate en torno a la atención de la salud mental. En este marco, se sancionaron las nuevas leyes de salud mental de la ciudad de Buenos Aires (448) en el año 2000 $y$ nacional (26657) en el año 2010. Estas normativas promueven un cambio de paradigma con respecto a la concepción sobre la salud mental, la forma de atenderla y el paciente. Ambas leyes apuntan a un pasaje de un modelo hospitalocéntrico -enfocado en la atención en hospitales monovalentes (los llamados manicomios) - a uno que privilegie prácticas ambulatorias. En términos prácticos esta normativa ordena la sustitución de los efectores monovalentes por dispositivos comunitarios que se adecúen a los principios de la ley, que fijó el año 2020 como plazo para la reconversión definitiva. Como veremos, esta obligación de sustituir los hospitales neuropsiquiátricos constituye uno de los argumentos a los que apela el GCBA para justificar el cierre de los de Borda y Moyano y la uti-

territorios $\overrightarrow{42}$ 


\section{$\Leftarrow$}

lización del predio donde funcionan para la radicación del distrito gubernamental.

19 Propuesta Republicana (PRO) es el partido politico conducido por el actual presidente de Argentina, Mauricio Macri. Este partido gobierna la ciudad de Buenos Aires desde 2007, teniendo como jefe de gobierno durante los primeros dos periodos a Mauricio Macri (2007-2015) $y$ actualmente a Horacio Rodriguez Larreta (2015 a la actualidad).

${ }^{20}$ Por falta de espacio, en este artículo solo se mencionan algunas generalidades en relación con esta progresiva politica de vaciamiento material del sistema de salud mental. Para conocer sus detalles ver Gonzalez Redondo (2018).

${ }^{21}$ Seguimos la propuesta teórico-metodológica que realiza esta autora para el análisis de los objetos discursivos en la medida en que la combinación de dos perspectivas teóricas: la de la escuela francesa y la de la lógica natural de Jean Blaise Grize, que permiten articular los niveles intere intradiscursivos.

${ }^{22}$ Esta publicación compila un diagnóstico oficial elaborado por la MCBA

territarios 42 son presentados en un relato que tiene una secuencia cronológica de pasado-quiebre o transformación-presente. Ambos aparecen en general como sujeto de nominalizaciones o en el lugar del objeto directo, en pasivo. Tanto a Barracas como al "sur" le pasan cosas. Barracas tenía un "carácter", una "identidad peculiar", un "rol protagónico", "sinónimo de progreso y desarrollo", que se interrumpe a partir de transformaciones tematizadas como "decadencia" o "desvanecimiento" que tienen como consecuencia la configuración de un presente "deteriorado" y "en estado de abandono". Así se presenta un pasado idílico que se contrapone a un presente valorado negativamente. Se plantea recurrentemente que la estrategia de intervención es "la recuperación de la identidad barrial", expresada también mediante sustitutos que presuponen un proceso de transformación: "recaracterización", "reformalización", "redefinición", "revitalización" y "rehabilitación". Asimismo, el uso de nominalizaciones como "la reconversión industrial", "la contaminación del Riachuelo" o "el inicio de la autopista" producen el efecto de sentido de ocultar los agentes de las acciones que producen dicho "deterioro".

$\mathrm{Al}$ igual que en el caso de Barracas, en estos discursos se sostiene que el predio de los hospitales "ha perdido sus características originales". Se lo tematiza como un "borde de fragmento urbano", "área en desuso", "deteriorada", "vacío urbano", "barrera urbana", "manzana en abandono total", que "configura un perímetro vacío de actividades y de significado". Como anticipamos, la repetición del oxímoron vacio urbano ${ }^{25}$ (o sustitutos) para caracterizar al predio de los hospitales es una constante en los documentos. Sin embargo, en cada caso este objeto discursivo adquiere nuevos sentidos y supone diversos implícitos. Así, las expresiones: barrera urbana y vacío urbano - ampliamente utilizadas en la retórica urbanística- aparecen tematizadas de la siguiente manera.

\section{Tabla 1. Tematización de las barreras y vacíos urbanos}

“impiden una relación fluida relación

$\mathrm{La}(\mathrm{s})$

barrera(s) entre las diversas áreas del mismo".

urbana(s) "significó un seccionamiento del tejido"

Los

"vacíos

urbanos"

"provocan la discontinuidad

del tejido urbano"

"atenta contra la posibilidad

integrativa que podría cumplir

como curso fluvial”

Fuente: Elaboración propia con base en el documento "Barracas. Diagnóstico y estrategias" (1992).

Como vemos en estas secuencias, la barrera urbana o el vacio urbano se colocan en el sujeto que lleva adelante la acción de ocasionar problemas a la integración del barrio. Particularmente se sostiene que los hospitales Borda, Moyano y Rawson

conforman barreras funcionales que no solo provocan discontinuidades en el tejido, sino que además albergan funciones que deterioran los entornos provocando la desvalorización de las tierras adyacentes y la

Carolina Gonzalez Redondo 
falta de inversiones tanto a nivel público como privado (MCBA, 1992, p. 14).

Entonces, de acuerdo con esta secuencia, los hospitales además de provocar discontinuidades en el "tejido", al igual que otras barreras urbanas, generan la desvalorización de las tierras y producen la falta de inversiones públicas y privadas. Resulta llamativa esta tematización que presentan los agentes, las acciones y las consecuencias de tal forma que en lugar de ser la falta de inversión pública la que deteriora estas "barreras" y por ende desvaloriza el entorno, son los hospitales los que deterioran el entorno y provocan la falta de inversión pública. A través de estas tematizaciones advertimos cómo se deslegitiman los usos y prácticas que se desarrollan en este espacio.

$\mathrm{Al}$ igual que se indica para el barrio de Barracas, la propuesta para el predio de los hospitales debe orientarse a recuperar ese pasado idílico, de ahí las nociones de "recaracterización", "reformalización" y "redefinición”. Así se reitera el lexema "recuperación" que produce un efecto de sentido metonímico: mientras que Barracas debe recuperarse para la ciudad, el predio de los hospitales debe recuperarse para el barrio.

\section{Área de desarrollo prioritario: una oportunidad para aprovechar}

En esta sección, analizamos documentos de diagnóstico y/o planificación que se elaboraron a partir del año 2000. En ellos advertimos un nuevo centramiento del objeto discursivo vacío urbano, que a su vez se apoya en un desplazamiento en el enunciador y enunciatario construidos en estos discursos. Estos son cambios graduales que también guardan relación con el contexto de producción de estas publicaciones. En lo que sigue, damos cuenta de estos desplazamientos en cada uno de los documentos, centrándonos en sus aspectos principales.

En el primer documento analizado - Relevamiento de áreas degradadas y áreas industriales obsoletas de la ciudad de Buenos Aires (2000)—, ${ }^{26}$ todavía identificamos un enunciador asociado a un ethos de experto, que es parte de una comunidad científica y de especialistas con quienes se comparte la mirada. Sin embargo, advertimos cómo este enunciador comienza a mezclarse con otro más cercano al empresario a partir de un ethos apoyado en un tono persuasivo, que produce un efecto se sentido de realce del barrio de Barracas y del área de los hospitales, destacando los atributos que son valorados positivamente. Este cambio en el tono del enunciador junto con un desplazamiento en la tematización del barrio y el predio en cuestión permiten suponer además la ampliación del enunciatario construido: ya no se dirige únicamente a los profesionales, técnicos y funcionarios públicos sino también a empresarios y a privados dispuestos a aprovechar las nuevas “áreas de oportunidad”. Este movimiento
$\Longleftarrow$

$y$ diferentes proyectos de intervención sobre áreas especificas de la ciudad, entre ellas, el predio de los hospitales y el barrio de Barracas.

${ }^{23}$ Este documento fue elaborado por el Programa de Revitalización Urbana del Sur de Buenos Aires (PROSUR) creado en 1990 a partir de un convenio entre la Secretaría de Planeamiento de la MCBA y Programa de Naciones Unidas para el Desarrollo (PNUD).

24 También observamos algunas diferencias $y$ desplazamientos entre estos documentos que, por cuestiones de espacio, no son abordadas en este articulo. Para profundizar sobre ello, consultar Gonzalez Redondo (2018).

25 Siguiendo a Stanchieri y Aricó (2013) sostenemos que la expresión vacio urbano constituye un oximoron - una combinación de dos términos que son contradictorios entre si-en la medida en que el espacio urbano nunca puede estar vacio. La definición misma de urbano implica vida, movimiento, relaciones sociales.

${ }^{26}$ Este documento fue elaborado en el año 2000 como producto de un convenio entre la FADU y la Secretaría de Planeamiento del GCBA.

tersitarios 42 
$\Longleftarrow$

La noción de estrategia tiene sus origenes en el ámbito militar y luego, a partir de los años 60, comienza a ser utilizada en los negocios. Dos décadas más tarde, la planificación estratégica pasó a formar parte del sentido común de la administración municipal estadounidense. Con el tiempo se tornó una fórmula exitosa que se implementó con diversos matices $y$ modalidades, en diferentes ciudades del mundo, Barcelona es uno de los casos más resonantes. Para profundizar en las premisas de la planificación estratégica urbana y su proceso de circulación ver Borja y Castells, 1997, Fernández Güell, 1997, Novais, 2010, $y$ Vainer, 2000.

${ }^{28}$ En el año 2000, se crea la Corporación Buenos Aires Sur, una sociedad del Estado a la que se le transfieren todos los inmuebles y terrenos públicos de dominio privado ubicados en el área de su competencia (el sur de la ciudad) para que los administre. Durante sus primeros años, la corporación llevó a cabo diversos proyectos en articulación con privados (el Centro Industrial del Sur; el Centro de distribución de medicamentos de Disprofarma; el Polo Farmacéutico, el Parque Roca) y

territarias 42

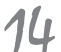

discursivo está en sintonía con los cambios de época, con las premisas que subyacen a las nuevas herramientas de planificación, como la planificación estratégica urbana, ${ }^{27}$ y con el tipo de gestión ligada al empresarialismo urbano (Harvey, 1989), en el que el Estado sale activamente a detectar ventajas competitivas para atraer capitales.

En coincidencia con documentos previos, la zona sur es aquí caracterizada como con "distintos niveles de degradación" y el agente de tal "degradación" es principalmente "el declive de la actividad industrial". Sin embargo, a partir de este documento advertimos la emergencia de un par que se asocia a "área degradada" que es la "zona o área de oportunidad" (o su sustituto "área de desarrollo prioritario"). Así, por ejemplo, la zona sur es caracterizada como un área "que encerraba junto con distintos niveles de degradación muchas oportunidades urbanísticas" (GCBA, 2000). Se sostiene, asimismo, que este pasaje de la "degradación" a la "oportunidad" implica un "necesario cambio", "un mejoramiento", "acciones puntuales de desarrollo", "rehabilitación", "promoción del desarrollo" y "áreas factibles de regenerar".

Advertimos, entonces, un nuevo centramiento del objeto discursivo vacio urbano que trae nuevos implícitos en relación con las estrategias de intervención en el predio de los hospitales. Mientras que en documentos previos al diagnóstico de “áreas degradadas", "vacíos urbanos" o "barreras urbanas" se lo asociaba a pro- puestas de "recuperación", "revitalización" y "rehabilitación", a partir de ahora comienza a aparecer más reiteradamente el lexema "desarrollar". En este sentido, a nivel propositivo el estudio introduce la noción de "área de desarrollo prioritario" en sintonía con la modificación realizada al Código de Planeamiento Urbano (CPU) unos meses más tarde que califica la zona sur como "área de desarrollo prioritario".

Estas "áreas de desarrollo prioritario" - entre las que se incluye al predio de los hospitales - son definidas como aquellas que "por su grado de obsolescencia, deterioro o subutilización se convierten en verdaderas oportunidades para ser desarrolladas por la vía promocional" (GCBA, 2000). En esta secuencia el uso del impersonal y del pasivo oculta el agente para el que suponen una oportunidad. Asimismo, la transición área "degradada" / "de oportunidad" / "de desarrollo prioritario" remite a diversos agentes en este documento: "el esfuerzo público", y en forma secundaria, "los vecinos a través de sus representantes naturales" y "la empresa privada". Así, junto con la zona de oportunidad aparece el rol del actor privado que hasta ahora no había sido incluido explícitamente en los otros documentos aquí analizados. Este cambio también está en sintonía con el contexto de producción del documento, en la medida en que es el momento en que están en curso o comienzan a concebirse (depende el caso) algunos proyectos urbanos en asociación público-privada bajo figuras jurídi- 
cas híbridas como la Corporación Puerto Madero y Corporación Buenos Aires Sur (Rodríguez, Bañuelos \& Mera, 2008). ${ }^{28}$

Puntualmente para el caso del predio de los hospitales, este pasaje implica un corrimiento en los atributos con los que se los tematiza. Si hasta aquí predominaba el uso de atributos negativos, a partir de este documento comienzan a sumarse algunas propiedades positivas, aquellas que hacen de un "área degradada", de un "espacio vacío", una "zona de oportunidad potencial significativa”. Así, en estos discursos, las estrategias de intervención se alejan de la idea de "recuperación" y comienzan a aparecer en forma más reiterada los lexemas: "renovación" y "desarrollo".

Este par “área degradada” / “área de oportunidad" se estabiliza en la publicación de 2009 que compila la ley 2930 del Plan Urbano Ambiental ${ }^{29}$ y el diagnóstico sobre el que se apoya. Este documento -al tratarse de una caracterización y una normativa de carácter general que cubre toda la ciudad en su inserción metropolitana- no menciona explícitamente al predio que aquí estudiamos, aunque sí se refiere a la zona sur, en la que se ubica este polígono. Advertimos que también en este documento se le asocian a la zona sur los siguientes atributos: "deterioro", “degradación”, “abandono", “contaminación", "vacancia". De la misma manera, la nominalización oculta los agentes que provocan tal deterioro. Ahora bien, en continuidad con el deslizamiento identificado a partir del documento anterior, observamos una apuesta por asociar este deterioro con un potencial o una oportunidad. De este modo, las expresiones "espacio vacío" y "espacio vacante" ya no están acentuadas negativamente, sino que pasan a connotar una "oportunidad".

\section{El "necesario" cambio de usos}

Los últimos dos documentos que analizamos son del año 2009 y presentan la propuesta del Parque Cívico (antecedente inmediato del distrito gubernamental) para "refuncionalizar" el predio de los hospitales. Uno de ellos, denominado "Proyecto y obras $2007 / 2009$ ", fue elaborado por la FADU y el Ministerio de Desarrollo Urbano (MDU) del GCBA. El otro - "Barracas. Diagnóstico y líneas de acción a escala barrial-", fue realizado por el Centro de Gestión y Participación Comunal 4 (CGPC) en conjunto con el mismo ministerio. A diferencia de los documentos previos, en estos dos casos el enunciador se posiciona en el rol de funcionario público asociado a una gestión de gobierno específica. Asimismo, se presenta el documento como "una herramienta técnico-política", como una "oportunidad política". Advertimos entonces en ambos casos un esfuerzo por asociar los diagnósticos y proyectos que allí se enumeran a una gestión política específica, algo que no sucede en los otros discursos analizados.

Desde este posicionamiento del enunciador, en "Barracas. Diagnóstico y líneas de acción a escala barrial" (2009) se sigue cretaron como los diferentes "polos" que pueden ser pensados como antecedente inmediato de los distritos especializados (Rodriguez, Bañuelos \& Mera, 2008; Arqueros Mejica, 2013).

${ }^{29}$ El Plan Urbano Ambiental constituye "la ley marco a la que deberá ajustarse la normativa urbanistica" (GCBA, 2009, p. 28). Su elaboración y aprobación fue producto de un largo proceso no exento de controversias. Ver Arqueros Mejica (2017).

territarias 42 
${ }^{30}$ La idea de crear una nueva centralidad administrativa, propia de la ciudad de Buenos Aires y diferenciada de la nacional, en la zona sur de la ciudad tiene su primer antecedente en el Plan Director (1938-1940), elaborado por Le Corbusier.

\section{territarias 42}

caracterizando al barrio como un área con problemas pero, al igual que señalamos en documentos previos, el énfasis está ahora en la idea de "oportunidad". Así la definición de los “problemas" estará siempre vinculada a su contracara: las "oportunidades" (o su sustituto "potencialidades"). Pero esas potencialidades están sujetas, en varios casos, a "cambios de usos" o "cambios de rubros".

Específicamente el predio de los hospitales también es caracterizado en este par problema/oportunidad: los hospitales “por su magnitud, dedicación y localización son generadores de barreras urbanísticas" y "conforman un enclave de magnitud relevante al poseer cerca de 38 has". Esta última secuencia, asociada a la tematización que se hace de estos terrenos, conlleva un implícito: se trata de una "magnitud relevante" para aprovechar, en definitiva, una oportunidad. Así, tal como lo advierten Stanchieri y Aricó (2013) el espacio vaciado e invisibilizado se transforma en un recurso. En sintonía con la propuesta de "cambio de usos" para otras zonas de Barracas, el proyecto para este predio es "descentralizar y relocalizar los usos de los hospitales" e "instalar en dicho espacio funciones del Gobierno de la Ciudad”. Ese enunciador posicionado en el rol de funcionario de "esta gestión política" presenta el Parque Cívico como una "propuesta concreta" a diferencia de las "sugerencias" plasmadas en documentos previos.

El documento "Proyecto y obras $2007 / 2009$ " (2010), por su parte, hace una presentación del proyecto del Parque Cívico. La preeminencia de modalidades como "la ciudad se merece" y "los vecinos tienen el derecho" tematizan a esta iniciativa como algo "necesario". Esa necesidad se ancla en dos motivos: la reformulación del sistema de salud mental y la autonomía de la ciudad de Buenos Aires. Si a un pasado dependiente ("poder delegado") le corresponde una "vieja Intendencia Municipal", un presente "autónomo" necesita de "otra estructura espacial". Así, el Parque Cívico es presentado como sinónimo de autonomía de la ciudad de Buenos Aires. ${ }^{30}$ Uno de los requisitos de esa autonomía sería una simbología propia, diferente de la nacional.

Como señalamos, el otro motivo que induce la "necesidad" de radicar el Parque Cívico en el predio en cuestión es el cambio en el paradigma de atención a la salud mental:

Si bien la localización seleccionada para el
nuevo Parque Cívico requiere de la relocali-
zación de algunas de las funciones existentes
en el predio, esta propuesta no es causa, sino
consecuencia del debate sobre el sistema
de Salud Mental que se merece la ciudad
y el cuestionamiento al sostenimiento de
un sistema que ya no se usa en el mundo
(GCBA, 2010, p. 21).

De esta secuencia se infiere, a diferencia de lo que sucede con documentos anteriores, que dicho predio no estaba "vacío" sino que alberga funciones, que 
son puestas en tela de juicio en la medida en que son parte de "un sistema que ya no se usa en el mundo". Asimismo el topos retórico (Amossy, 2000) "no es causa, sino consecuencia" produce el efecto de sentido de corregir una afirmación que sostiene lo contrario. Así, se aclara y se infiere que el debate sobre el sistema de salud mental tiene como consecuencia natural, necesaria y única la liberación de esos terrenos y su refuncionalización para un Parque Cívico. Quedan vedados así otros posibles usos de esos terrenos.

Estos dos documentos continúan sosteniendo ese pasaje de "espacio vacío" a “área de oportunidad”. Pero introducen más concretamente que para que ese pasaje se haga efectivo, para que esa oportunidad se aproveche es preciso "cambiar de usos". Para el predio en cuestión ese "cambio de usos" significará relocalizar las funciones de los hospitales e instalar allí el Parque Cívico, una nueva estructura cívica que "merece" y "necesita” la ciudad para afianzar su autonomía. ${ }^{31}$ Se trata de un punto de quiebre con respecto a propuestas previas que buscaban "rehabilitar", "recalificar" y "revitalizar" dicho espacio. A partir de este momento, se pone por primera vez en discusión el cierre o relocalización de estos hospitales.

\section{Reflexiones finales}

A lo largo de este trabajo analizamos cómo la recurrente problematización del predio de los hospitales como un "espacio

EL VACIAMIENTO QUE ANTECEDE LA RENOVACIÓN vacío" (o sustitutos) constituye el asunto que el poder ejecutivo local —con diferentes matices y en distintos momentos en el tiempo- transforma en cuestión (Oszlak y O'Donnell, 1981) y, por tanto, justifica y legitima la creación del distrito gubernamental como una política pública tendiente a "desarrollar" esa zona "deteriorada" del barrio de Barracas. Así, dimos cuenta de cómo se produjo un proceso de vaciamiento simbólico, sostenido en discursos que constituyen miradas oficiales sobre la ciudad, que resulta condición para el despliegue de esta política de renovación urbana.

A través del análisis discursivo advertimos cómo la propia noción de "renovación" fue adquiriendo relevancia a través de los distintos centramientos del objeto discursivo: vacío urbano. En un primer momento, la tematización del presente de Barracas y del predio de los hospitales como áreas "deterioradas" y "vacías" se contrapone con un pasado idílico al que era preciso retornar. Así las propuestas de intervención buscaban transformar estos espacios mediante procesos de "recaracterización", "reformalización", "redefinición", "revitalización”, etc.

A partir del año 2000, en sintonía con los nuevos modelos de gestión urbana, cambia la tematización del barrio y del predio. Al "área deteriorada” y "espacio vacío" se le comienzan a asociar las expresiones "área de oportunidad" y "área de desarrollo prioritario". Este movimiento discusivo se apoya en un cambio en la
31 Recordemos también que este es un contexto en que las gestiones de la ciudad de Buenos Aires y de la Nación eran de signo politico distinto y manifestaban por entonces fuertes discrepancias. territarios 42

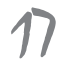


figura del enunciador, que combina su rol de experto con un tono persuasivo, más cercano al rol del empresario, y en una ampliación del enunciatario construido, que además de contemplar expertos y funcionarios públicos suma actores privados dispuestos a aprovechar las nuevas “áreas de oportunidad”. De este modo, vemos cómo el urbanismo neoliberal y el empresarialismo urbano se expresan también en los documentos normativos que proponen una mirada y una intervención sobre la ciudad. Es el propio Estado en su rol de facilitador quien, a través de un enunciador de experto y empresario, identifica los "espacios vacíos" y los presenta como una "oportunidad" para que el privado desarrolle.

A partir de 2009, se continúa acentuando la idea de oportunidad, pero se refuerza con un "necesario cambio de usos" para que la misma sea aprovechada. Así, este espacio vaciado se transforma en un recurso. Sin embargo, para que efectivamente aparezcan las propuestas de renovación, es necesario que este predio se vacíe materialmente, de ahí que el proceso de vaciamiento simbólico corra en paralelo a la política de vaciamiento material: proceso de destrucción creativa de dicho predio.

Por otro lado, cabe señalar que las condiciones de aparición de un objeto discursivo - en este caso, el vacío urbano- son históricas y exceden al sujeto-enunciador. De ahí la importancia de reconstruir el territarias 42 18 del distrito gubernamental y, a la vez, dar cuenta del proceso histórico de urbanización de Buenos Aires, reponiendo los agentes del "deterioro" y del "desequilibrio" que en los discursos analizados aparecen borrados.

El análisis discursivo aquí desplegado contribuye a dar cuenta de los modos específicos en que se vacían simbólicamente los espacios urbanos para luego poder presentar los proyectos de (re)llenado y dar lugar a las políticas de renovación. También permite analizar críticamente discursos muy consolidados y de carácter constituyente como el científico y específicamente el urbanismo. En este sentido, el abordaje discursivo de la política urbana resulta productivo para reflexionar en torno a la dimensión del espacio concebido (Lefebvre, 2013) y a su fuerza performativa en la producción del espacio urbano.

\section{Referencias}

Amossy, R. (2000). Lo plausible y lo evidente: doxa, interdiscurso, tópicos. En L'argumentation dans le discours. Discours politique, littérature d'idées, fiction (pp. 1-25). París: Nathan.

Angenot, M. (2010). El discurso social: problemática de conjunto. In $E l$ discurso social. México: Siglo XXI Editores.

Arqueros Mejica, M. S. (2013). Procesos de produción social del hábitat y politicas públicas en las villas de la Ciudad de Buenos Aires. El caso del Barrio INTA. (Tesis de maestría, Universidad 
Torcuato Di Tella, Buenos Aires, Argentina).

Arqueros Mejica, M. S. (2017). La politica de desarrollo de la Comuna 8 de la ciudad de Buenos Aires (1996-2015). (Tesis de doctorado, Universidad de Buenos Aires, Argentina).

Arqueros Mejica, M. S. et al. (2011). Apuntes sobre la cuestión villera en la ciudad de Buenos Aires. Revista Herramienta, (48). Recuperado de http://www.herramienta.com.ar/ print/revista-herramienta-n-48/ apuntes-sobre-la-cuestion-villera-enla-ciudad-de-buenos-aires

Borja, J. \& Castells, M. (1998). Local y global. La gestión de las ciudades en la era de la información. Buenos Aires: Taurus

CESLESM (2010). Informe de seguimiento. CESLESM. (2012). Informe de seguimiento. CESLESM. (2015). Informe de seguimiento bianual.

CEyS (2013). Diagnóstico Socio-habitacional de la ciudad de Buenos Aires. Buenos Aires: GCBA.

Charaudeau, P. \& Mainguenau, D. (Dirs.) (2005) Diccionario de análisis del discurso, Buenos Aires: Amorrortu Ediciones.

Di Virgilio, M. \& Guevara, T. (2014). Gentrificación liderada por el Estado y empresarialismo urbano en la Ciudad Autónoma de Buenos Aires. Revista Estudios Sociales Contemporáneos, (11), $12-23$.

EL VACIAMIENTO QUE ANTECEDE LA RENOVACIÓN
Díaz Parra, I. (2013) La gentrificación en la cambiante estructura socioespacial de la ciudad. Revista Bibliográfica de Geografia y Ciencias Sociales, 18(1030). Recuperado de http://www.ub.edu/ geocrit/b3w-1030.htm

Fernández Güell, J. M. (2007). Planificación Estratégica de Ciudade. Barcelona: Editprial Gustavo Gili.

Foucault, M. (2002). La arqueología del saber. Buenos Aires: Siglo XXI Editores.

Franquesa, J. (2007). Vaciar y llenar, o la lógica espacial de la neoliberalización. Reis: Revista Española de Investigaciones Sociologicas, (118), 123-150. Doi: https://doi.org/10.2307/40184799 GCBA (2000) Relevamiento de Áreas Degradadas y Áreas Industriales Obsoletas de la Ciudad de Buenos Aires. Buenos Aires.

GCBA. (2009). Ley No2930 Plan Urbano Ambiental.

GCBA. (2009b). Barracas. Diagnóstico y líneas de acción a escala barrial. Buenos Aires GCBA. (2010). Proyecto y obras 2007/2009. Buenos Aires.

Goicoechea, M. E. (2016). Distritos creativos en el sur de la ciudad de Buenos Aires (2008-2015). Renovación urbana y nuevas lógicas de segregación, 16(9), 224-227.

Gonzalez Redondo, C. (2015). Desarrollar el sur: el caso del distrito gubernamental en la Ciudad de Buenos Aires. Letra. Imagen. Sonido, 7(14), 181-200.

Gonzalez Redondo, C. (2018). Del distrito gubernamental a la nueva sede de territarias 42

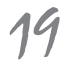




\section{territarias 42}

gobierno. Disputas en torno al espacio urbano en la ciudad de Buenos Aires. (Tesis de maestría, Universidad de Buenos Aires, Argentina).

Gorelik, A. (1998). La grilla y el parque. Espacio público y cultura urbana en Buenos Aires, 1887-1936. Buenos Aires: Universidad Nacional de Quilmes.

Gorelik, A. (2004). Miradas sobre Buenos Aies. Historia cultural y critica urbana. Buenos Aires: Siglo XXI Editores.

Guevara, T. (2010). Politicas habitacionales y procesos de producción del bábitat en la Ciudad de Buenos Aires. El caso de La Boca. (Tesis de maestría, Universidad de Buenos Aires, Argentina).

Harvey, D. (1989). From managerialism to entrepreneurialism: the transformation in urban governance in late capitalism. Geografiska Annaler. Series B, Human Geography, 71(1), 3-17. Doi: https:// doi.org/10.2307/490503

Herzer, H. (2008). Acerca de la gentrificación. En Con el corazón mirando al sur. Buenos Aires: Espacio.

Jajamovich, G. (2012). La ciudad en cuestión. Pugnas y reconfiguraciones de redes de técnicos y profesionales que intervienen sobre la ciudad de Buenos Aires (1983-1992). (Tesis de doctorado, Universidad de Buenos Aires, Argentina).

Jajamovich, G. \& Menazzi, L. (2012). Políticas urbanas en un contexto de dictadura militar: algunos interrogantes a partir de Buenos Aires (1976-1983).
Bitácora Urbano Territorial, 20(1), 11-20.

Jaramillo, S. (2014). Acerca de la investigación en mercados de tierra urbana. En B. R. Ramírez Velázquez \& E. Pradilla Cobos (Comps.) Teorías sobre la ciudad en América Latina. México: Universidad Autónoma Metropolitana. Lefebvre, H. (2013). La producción del espacio. Madrid: Capitán Swing.

Maingueneau, D. (2008). Términos clave del análisis del discurso. Buenos Aires: Nueva Visión.

Maingueneau, D. \& Cossutta, F. (1995). L'analyse des discourse constituans. Langages, (117), 112-125.

Maldidier, D. (1990). La inquietud del discurso. Un trayecto en la historia del análisis del discurso. El trabajo de Michel Pêcheux. Signo \& Seña, (1), 201-213.

Marcús, J., Aquino, M. de la P., \& Vázquez, D. E. (2016). Espacios urbanos vaciados, proyectos de renovación urbanística y resistencia vecinal en la Ciudad de Buenos Aires: el caso de la manzana 66 del barrio de Balvanera. Quid 16. Revista Del Área de Estudios Urbanos, (6), 253-280.

Marcús, J. \& Vázquez, D. (2017). Vacias, llenar, resistir. Conflictos y tensiones en la producción de "vacíos urbanos". En J. Marcús (Coord.), Ciudad Viva. Disputas por la producción sociocultural del espacio urbano en la ciudad de Buenos Aires. Buenos Aires: Teseo. 
de Mattos, C. (2008). Globalización, negocios inmobiliarios y mercantilización del desarrollo urbano. En M. Córdova Montúfar (Coord.), Lo urbano en su complejidad: una lectura desde América Latina. (pp. 35-62). Quito: Flacso.

Montero, A. S. (2014). El análisis francés del discurso y el abordaje de las voces ajenas: interdiscurso, polifonía, heterogeneidad y topos. En M. Canales (Comp.), Escucha de la escucha. Análisis e interpretación en la investigación cualitativa (pp. 247-273). Santiago de Chile: LOM-FACSO.

Narvaja de Arnoux, E. (2009). Análisis del discurso. Modos de abordar materiales de archivo. Buenos Aires: Santiago Arcos.

Novais, P. (2010). Uma estratégia chamada "Planejamento estratégico". Río de Janeiro: 7 Letras Editorial.

Oszlak, O. (1983). Los sectores populares y el derecho al espacio urbano. SCA Revista de La Sociedad Central de Arquitectos, (125), 1-7.

Oszlak, O. (2017). Merecer la ciudad. Buenos Aires: UNTREF.

Oszlak, O. \& O’Donnell, G. (1981). Estado y políticas estatales en América Latina: hacia una estrategia de investigación. Cedes, (4), 98-128.

Pírez, P. (1995). Actores sociales y gestión de la ciudad. Ciudades, (28), 1-12.

Pradilla Cobos, E. (2009). Las políticas y la planeación urbana en el neoliberalismo. En P. Brand (Ed.), La ciudad latinoamericana en el siglo XXI. Glo- balización, neoliberalismo, planeación (pp. 287-307). Medellín: Universidad Nacional de Colombia. Recuperado de http://www.emiliopradillacobos. com/TexABR2011/las politicas y la planeacion urbana en el neoliberalismo.PDF

Pradilla Cobos, E. \& Márquez López, L. (2016). Los territorios latinoamericanos en la mundialización del capital. Territorios, (34), 17-34.

Rodríguez, M. C, Bañuelos, C., \& Mera, G. (2008). Intervención - no intervención: ciudad y políticas públicas en el proceso de renovación del Área Sur de la Ciudad de Buenos Aires. En H. Herzer (Org.), Con el corazón mirando al sur. Transformaciones en el sur de la ciudad de Buenos Aires (pp. 45-92). Buenos Aires: Espacio Editorial.

Rodríguez, M. C. (2015). Estado, clases sociales y gentrificación. La política urbana como campo de disputa en tres barrios de la Ciudad de Buenos Aires. En V. Delgadillo, I. Díaz Parra \& L. Salinas (Coords.), Perspectivas del estudio de la gentrificación en México y América Latina (pp. 205-227). México: UNAM.

Rodríguez, M. C. \& Di Virgilio, M. (2014). Ciudad de Buenos Aires: políticas urbanas neoliberales, transformaciones socio-territoriales y hábitat popular. Revista de Direito da Cidade, 6(2), 323.347. Doi: https://doi. org $/ 10.12957 /$ rdc.2016.19115 territarios 42 21 
Romero, J. L. (2009). Buenos Aires: una historia. En La ciudad occidental. Buenos Aires: Siglo XXI Editores.

Scobie, J. (1977). Buenos Aires. Del centro a los barrios 1870-1910. Buenos Aires: Ediciones Solar/Hachette.

Silvestri, G. \& Gorelik, A. (2000). Ciudad y cultura urbana (1976-1999): el fin de la expansión. En J. L. Romero \& L. A. Romero (Dirs.), Buenos Aires. Historia de cuatro siglos (pp. 461-499). Buenos Aires: Altamira.

Smith, N. (2012). La nueva frontera urbana. Ciudad revanchista y gentrificación. Madrid: Traficantes de sueños. Recuperado de https://www.traficantes. net/sites/default/files/pdfs/La\%20 nueva\%20frontera\%20urbana-TdS.pdf
Stanchieri, M. L. \& Aricó, G. (2013). La trampa urbanistica de los "vacios urbanos": casos etnográficos en Barcelona. X Jornadas de Sociología de la UBA, (1 al 6 de julio de 2013, Buenos Aires). Subirats, J., Knoepfel, P., Larrue, P., \& Varonne, F. (2008). Análisis y gestión de politicas públicas. Barcelona: Ariel.

Theodore, N., Peck, J., \& Brenner, N. (2009). Urbanismo neoliberal: la ciudad y el imperio de los mercados. Temas Sociales SUR, (66), 1-11.

Vainer, C. (2000). Pátria, empresa e mercadoria. Notas sobre a estratégia discursiva do Planejamento Estratégico Urbano. En O. B. Arantes, C. Vainer \& E. Maricato (Eds.), A cidade do pensamento único. Río de Janeiro: Editora Vozes. 